\title{
Algoritmos Bioinspirados - Uma Revisão Sistemática da Literatura no Brasil
}

\author{
Lucas Leal Soares ${ }^{1}$, Vinícius Martins Tork ${ }^{1}$, Wesley Gabriel Pereira Gomes ${ }^{1}$, Alan \\ Marcel Fernandes de Souza ${ }^{1}$, Polyana Santos Fonseca Nascimento ${ }^{1}$, Roberto Célio \\ Limão de Oliveira ${ }^{2}$
}

${ }^{1}$ ARGO - Centro Universitário do Estado do Pará (CESUPA) - 66040-020 - Belém, PA - Brasil

${ }^{2}$ Faculdade de Engenharia da Computação e de Telecomunicações - Universidade Federal do Pará - 66075-110 - Belém, PA - Brasil.

\{lucas17070019,vinicius17070014, wesley17070015\} @aluno. cesupa.br, \{alan.souza, polyana.nascimento\}@prof.cesupa.br, limao@ufpa.br

\begin{abstract}
This paper presents a systematic literature review of works referent to bioinspired algorithms published in Brazil. In this study, the repositories of scientific works from the federal universities of Brazil were used to research graduation final papers, master's degree dissertations and doctorate degree dissertations published between 2016 and 2020 that involved the cited thematic. To this end, the following keywords were utilized in the search: "bioinspirado", "metaheurístico" and "inteligência artificial”. Analyzing the collected data, it was noted that the amount of works about bioinspired algorithms was small, an indication that the field of research was poorly explored in Federal Universities of Brazil.
\end{abstract}

Resumo. Este artigo apresenta uma revisão sistemática da literatura publicada no Brasil referente aos algoritmos bioinspirados. Neste estudo, os repositórios de trabalhos científicos das universidades federais do Brasil foram usados para pesquisar trabalhos de conclusão de curso, dissertações de mestrado e teses de doutorado publicadas entre os anos de 2016 a 2020. Para isso, utilizou-se as seguintes palavras-chave na busca: "bioinspirado", "metaheurístico" e "inteligência artificial". Ao analisar os dados coletados, notou-se que a quantidade de trabalhos sobre algoritmos bioinspirados é pequena, sendo um indicio de que este campo de pesquisa ainda é pouco explorado nas Universidades Federais do Brasil.

\section{Introdução}

A computação natural é um ramo da ciência da computação destinado a estudar, compreender e aplicar, padrões complexos encontrados na natureza, utilizando-os como base para resolução de problemas, desenvolvimento de novas tecnologias e aperfeiçoamento de sistemas já existentes [De Castro 2007]. Uma das suas especializações é a computação bioinspirada, que visa estudar os comportamentos observados na natureza e aplicá-los na computação para a resolução de problemas computacionais, dentre os quais: movimentação de robôs, classificação de dados, 
reconhecimento de padrões em big data e criação de dispositivos que emulam o comportamento animal para fins de estudo [Xin-She et. al. 2013].

Portanto, a computação natural, sendo uma área de pesquisa em crescimento, é capaz de propor novas possibilidades de resolução de problemas de forma a trazer novos paradigmas na computação, podendo substituir os métodos tradicionais de resolução de problemas. A partir dessa premissa, este artigo tem como objetivo realizar uma revisão sistemática da literatura sobre algoritmos bioinspirados nos repositórios públicos das universidades brasileiras, avaliando deste modo o progresso da área de 2016 a 2020. Apenas repositórios das universidades públicas das capitais foram usados, porque nessas instituições há investimentos em pesquisa e somente trabalhos do período citado foram considerados, pois o foco foi em pesquisas recentes.

Sendo assim, um banco de dados de trabalhos acadêmicos foi gerado a partir da pesquisa e foi realizado um estudo sobre esses trabalhos na área de algoritmos bioinspirados, com o intuito de avaliar a relevância dos mesmos para a comunidade científica com a finalidade de verificar se a área necessita ser mais incentivada e explorada no país.

Além da introdução, este artigo está dividido em mais quatro seções: metodologia, resultados e discussão, conclusão e referências.

\section{Metodologia}

A metodologia utilizada para realizar a revisão sistemática deste trabalho - baseada em Souza (2020) e sumarizada no quadro 1 - consistiu em definir a pergunta inicial de pesquisa e depois, os termos de busca foram estabelecidos a partir da pergunta. $\mathrm{O}$ próximo passo foi fazer a pesquisa em si, salvando os trabalhos que satisfazem a pergunta em um banco de dados de trabalhos \#1.

Quadro 1. Parâmetros de pesquisa

\begin{tabular}{|l|l|}
\hline \multicolumn{1}{|c|}{ Parâmetro } & \multicolumn{1}{c|}{ Valor } \\
\hline $\begin{array}{l}\text { Pergunta de pesquisa } \\
\# 1\end{array}$ & $\begin{array}{l}\text { Qual o panorama dos trabalhos acadêmicos que utilizam } \\
\text { algoritmos bioinspirados baseado principalmente em } \\
\text { animais/insetos para resolução de problemas de } \\
\text { otimização? }\end{array}$ \\
\hline Termos de busca & bioinspirado, metaheurístico, inteligência artificial \\
\hline $\begin{array}{l}\text { Pergunta de pesquisa } \\
\text { \#2 }\end{array}$ & $\begin{array}{l}\text { Dos trabalhos que utilizam algoritmos bioinspirados para } \\
\text { resolução de problemas de otimização, quais deles } \\
\text { envolvem o algoritmo ACO (Ant Colony Optimization }) ?\end{array}$ \\
\hline Ano de publicação & De janeiro/2016 a dezembro/2020 \\
\hline Idioma & Português ou Inglês \\
\hline
\end{tabular}

Posteriormente, aplicaram-se critérios de rejeição nesse primeiro banco de dados. Como a primeira pergunta é muito abrangente, então o passo seguinte foi a realização de uma segunda pergunta de pesquisa mais específica, gerando um banco de trabalhos \#2. Nesse banco, analisaram-se os títulos e resumos de cada trabalho, listando detalhes dos mesmos. Encontrado os trabalhos que satisfazem a segunda pergunta, foi feita um resumo desses trabalhos e a adição dos memos nos resultados. A figura 1 sumariza a metodologia usada neste artigo. 


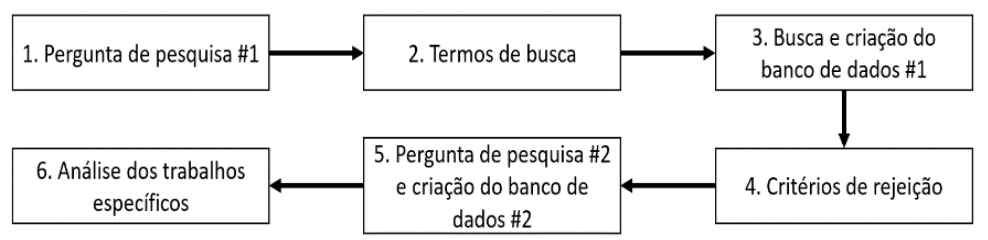

Figura 1. Fluxograma da revisão sistemática.

Neste trabalho, repositórios de trabalhos acadêmicos das universidades federais do Brasil (apenas capitais e uma universidade por capital) foram usados nas pesquisas, especificamente os repositórios próprios das universidades, por exemplo: http://repositorio.ufpa.br/ e https://repositorio.usp.br/. No total, 45 trabalhos foram préselecionados e inseridos no banco de dados \#1. Após as filtragens, o banco de dados \#2 foi gerado que considera seis trabalhos.

\section{Resultados e discussão}

Após a execução da metodologia descrita, os resultados foram gerados.

De acordo com o gráfico da figura 2, observa-se um aumento no número de produções de 2016 a 2018, porém, em 2019, verifica-se uma queda no número de produções científicas nessa área, em 2020 nenhuma produção nessa área foi desenvolvida até o mês no qual foi desenvolvida a pesquisa (mês de setembro/2020). Em grande parte, devido a pandemia causada pelo Covid-19, não foram publicados trabalhos no ano de 2020, devido as medidas sanitárias recomendadas, o departamento responsável por disponibilizar os trabalhos nos repositórios não têm funcionado. No total, considerando os últimos quatro anos, há 45 trabalhos publicados. A queda registrada de 2018-2019 pode ter sido causada pela falta de incentivo na pesquisa (cortes de verbas) [Escobar 2020].

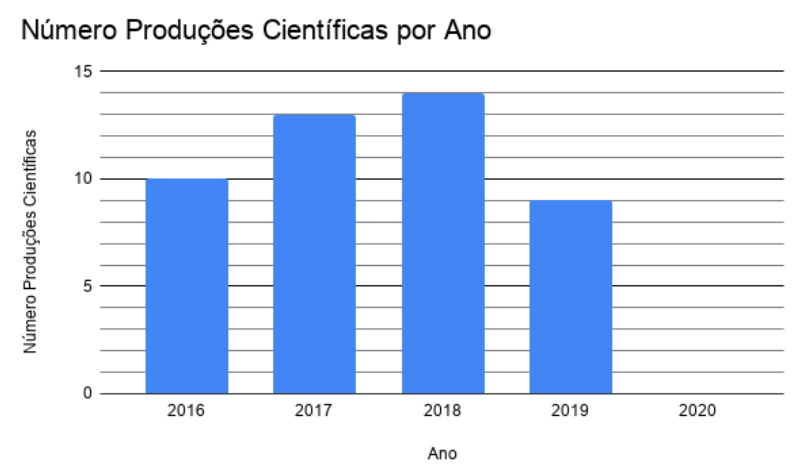

Figura 2. Gráfico com número de produções científicas por ano.

Conforme a figura 3 , podemos analisar que a concentração das pesquisas nessa área está nas regiões nordeste e sudeste, isso deve-se à concentração de polos de tecnologia localizados em estados como São Paulo, Rio de Janeiro e Pernambuco. Porém, a região nordeste conseguiu se destacar mais dentro da área devido ao processo de crescimento econômico que a região passou há alguns anos [IBGE 2021]. Outro ponto evidenciado é o fato de o centro-oeste ser a região com menos produções e o Pará liderar o quantitativo de pesquisas na região norte. 


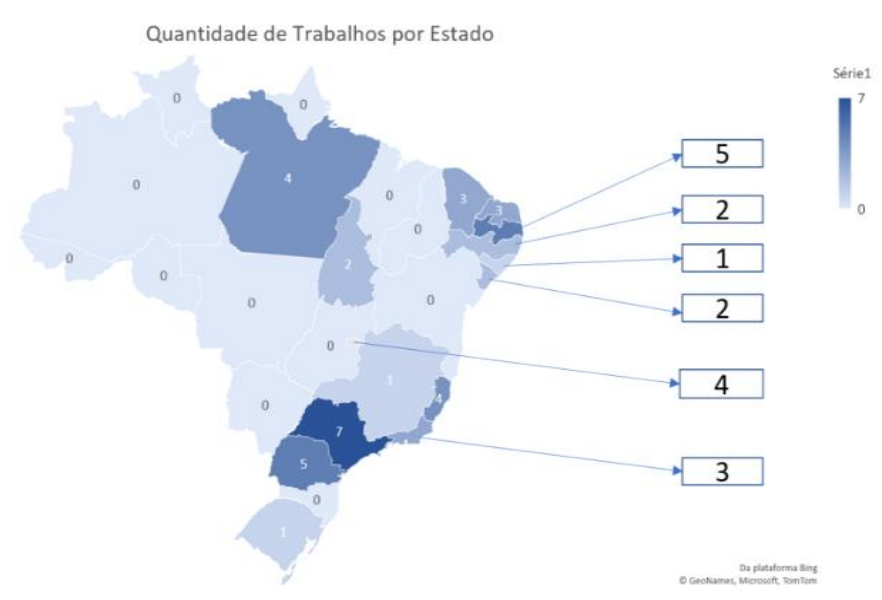

Figura 3. Gráfico com número de produções científicas por regiões.

\section{Conclusão}

A revisão sistemática da literatura descrita neste trabalho, que considerou os repositórios de universidades federais brasileiras e trabalhos publicados entre 2016 e 2020, atestou que as regiões sul e sudeste do país concentram a maior parte dos trabalhos publicados na área em pauta. Além disso, pela baixa quantidade de trabalhos, verifica-se que os algoritmos bioinspirados são pouco explorados em trabalhos de conclusão de curso, dissertações de mestrado e teses de doutorado que são disponibilizadas nos referidos repositórios.

Como trabalhos futuros, pretende-se expandir os repositórios para outras universidades públicas e privadas do Brasil. Ademais, aspira-se realizar comparações entre repositórios nacionais e estrangeiros.

\section{Referências}

De Castro, L. N., Fundamentals of Natural Computing: Basic Concepts, Algorithms, and Applications, Boca Raton, FL: CRC Press, 2007.

Escobar, E. Orçamento 2021 condena ciência brasileira a "estado vegetativo". Disponível em: https://jornal.usp.br/universidade/politicas-cientificas/orcamento2021-coloca-ciencia-brasileira-em-estado-vegetativo/. Acessado em outubro de 2021.

IBGE. Contabilidade Nacional e o Nordeste - IBGE. Disponível em: https://agenciadenoticias.ibge.gov.br/media/com_mediaibge/arquivos/7259be51a5b8 41ae50b810fc3b948ebf.pdf. Acessado em outubro de 2021.

Souza, A. M. F. de, Uso de Técnicas de Aprendizado de Máquina para Extração de Conhecimento e Modelagem do Processo de Produção de Alumínio Primário. $131 \mathrm{f}$. 2020. Tese (Doutorado no Programa de Pós-Graduação em Engenharia Elétrica) Instituto de Tecnologia, Universidade Federal do Pará, Belém, 2020.

Xin-She Yang, Zhihua Cui, Renbin Xiao, Amir Hossein Gandomi, Mehmet Karamanoglu (Editors). Swarm Intelligence and Bio-Inspired Computation: Theory and Applications, Elsevier, 2013. 